Haben Sie Fragen zur Abrechnung oder zur wirtschaftlichen Praxisführung? Als Leser der MMW können Sie sich an unseren Experten wenden: Helmut Walbert, Facharzt für Allgemeinmedizin und Betriebswirt aus Würzburg.

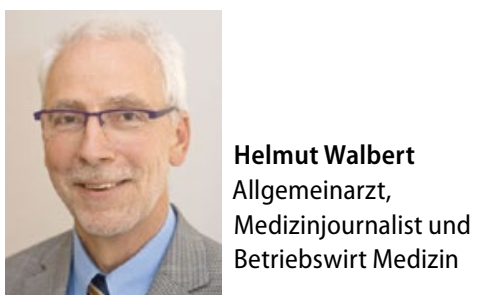

\title{
Arbeitsunfall
}

\section{Die BG zahlt auch, wenn es „Ihr" Patient ist}

$?$ Dr. F.-.J. Z., Landarzt, Allgemeinarzt, Nordrhein: Ich wurde aus der Sprechstunde heraus zu einem Arbeitsunfall auf einer Baustelle gerufen. Der Verletzte ist Patient bei mir. Kann ich Leistungen wie Infusion oder Verbände abrechnen? Vieles fällt ja eigentlich in die Hausarztpauschale.

MMW-Experte Walbert: Die Tatsache, dass der Unfallverletzte ein Patient der Praxis ist, hat lediglich die Datenerfassung erleichtert. Hier liegt eindeutig ein Arbeitsunfall vor, und dieser wird grundsätzlich über die zuständige Berufsgenossenschaft (BG) abgerechnet. Das gilt auch für die Erst- bzw. Notfallversorgung durch einen Hausarzt. Abgerechnet wird nach der mit den Unfallversicherungsträgern vereinbarten Gebührenordnung, der UVGOÄ. Diese weicht von der normalen GOÄ sehr stark ab, sowohl bei den Ziffern als auch in der Bewertung. Im Internet gibt es den Text etwa unter www.kbv.de/media/ sp/UV_GOAE.pdf.
Die Liquidation wird an die zuständige BG gestellt. Neben den Personaldaten sind der Arbeitgeber und die Unfallstelle sowie der Unfallzeitpunkt anzugeben. Entsprechende Formulare sind in den meisten Praxisverwaltungssystemen vorhanden. Wenn nicht: Anruf bei der nächsten $B G$, die ein entsprechendes Formular zuschickt. Jede BG gibt ggf. auch gern Auskunft, wie man erbrachte Leistungen wie Infusionen oder verbrauchtes Material abrechnet.

Für die Notfall- oder Erstversorgung von BG-Unfällen bedarf es keiner besonderen Qualifikation. Vertragsärzte können Arbeits-, Schul- oder Wegeunfälle problemlos in der Hausarztpraxis erstversorgen oder nach Zurücküberweisung durch den Unfall- oder D-Arzt zulasten der BG weiterversorgen. Daher sollte auch bei Bagatell- oder Kleinunfällen immer nachgefragt werden, bei welcher Tätigkeit der Schaden entstanden ist. Ein Unfall beim Einkauf für eine erkrankte Nachbarin z. B. ist ein Wegeunfall, der

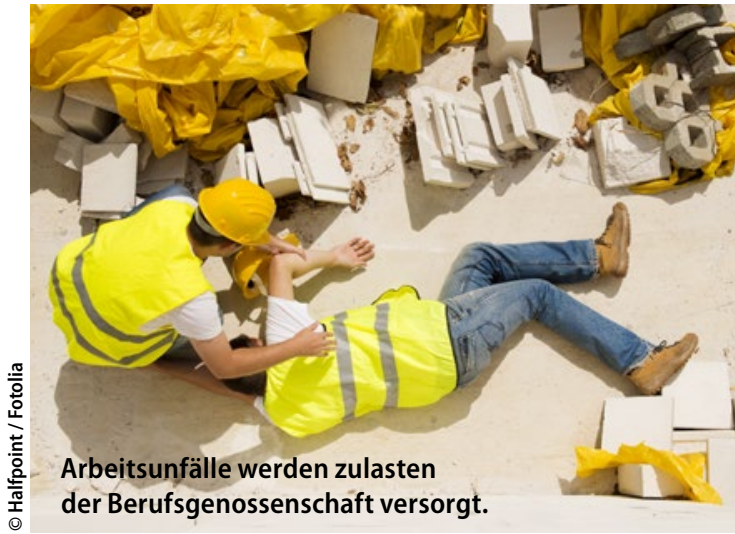

zulasten der BG Gemeinde-Unfallversicherung versorgt wird.

Bei der Frage, ob es denn ein Unfall war, sollte man erst einmal "großzügig“ sein, solange man den Hergang möglichst exakt darstellt. Die BG kann den Unfall ja auch ablehnen. Die Kosten für Meldung und Erstversorgung werden meist dennoch anstandslos übernommen, was ein Honorar außerhalb der budgetierten Vertragsarzttätigkeit darstellt.

\section{MFA besucht Privatpatienten}

\section{Alle Delegationsleistungen nach GOÄ abrechnen}

a O. G., leitende MFA in einer Familienpraxis, Bayern: Bei uns werden regelmäßig Hausbesuche durch Medizinische Fachangestellte (MFA) gemacht - auch bei Privatpatienten. Was kann neben der GOP 52 an Leistungen abgerechnet werden?
! MMW-Experte Walbert: Grundsätzlich können bei einem Besuch durch nichtärztliches Personal nach GOP 52 alle delegationsfähigen Leistungen abgerechnet werden. Wichtig ist, dass die MFA für die jeweilige Leistung qualifiziert ist. Angefangen bei diagnostischen
Maßnahmen wie EKG, Lungenfunktion und Demenzteste über Injektionen und Infusionen bis hin zur Dekubitus- und Ulkusbetreuung inklusive Verbände sind alle Delegationsleistungen abrechenbar. Hinzu kommen die nach $\$ 10$ der GOÄ berechnungsfähigen Auslagen. 